\title{
Selective Attention and Information Processing Speed in Graves’ Disease: Stroop Interference Effect
}

İskender Ekinci ${ }^{1}$, Esra Ekinci ${ }^{2}$, Mitat Buyukkaba ${ }^{1}$, Ahmet Cinar ${ }^{3}$, İrem Kirac Utku ${ }^{4}$, Hanise Ozkan ${ }^{4}$, Muhammed Tunc ${ }^{5}$, Abdulbaki Kumbasar ${ }^{1}$, Omur Tabak ${ }^{1}$

1. Internal Medicine, Health Sciences University, Kanuni Sultan Suleyman Training and Research Hospital, Istanbul, TUR 2. Social Services Department, Directorate of Woman and Family Services, Istanbul Metropolitan Municipality, Istanbul, TUR 3. Internal Medicine, Arnavutkoy State Hospital, Istanbul, TUR 4. Internal Medicine, Kanuni Sultan Suleyman Training and Research Hospital, Istanbul, TUR 5. Internal Medicine, Bezmialem Vakif University, Istanbul, TUR

Corresponding author: İskender Ekinci, driskenderekinci@gmail.com

\section{Abstract \\ Background}

Cognitive functions are affected by thyroid hormones. In this study, we aimed to investigate the selective attention and information processing speed in thyrotoxic Graves' disease.

\section{Methodology}

This study was conducted among 40 patients with thyrotoxic Graves' disease and age and gender-matched 40 healthy controls. Stroop Color and Word test were applied to healthy controls once and to patients with Graves' disease during thyrotoxic and euthyroid periods. Stroop interference effect was calculated.

\section{Results}

The mean age was $34.67 \pm 11$ in the Graves' group and $34.72 \pm 9.16$ in the control group $(p>0.05)$. The number of errors and self-corrections in Stroop Color and Word test was higher in patients with thyrotoxic Graves' disease than both patients with euthyroid Graves' disease and healthy controls $(p<0.05)$. Stroop interference effect was significantly longer in patients with thyrotoxic Graves' disease than both patients with euthyroid Graves' disease and healthy controls $(p<0.05)$. All parameters obtained from the Stroop Color and Word test including errors, self-corrections, and Stroop interference effect were similar in patients with euthyroid Graves' disease and healthy controls.

\section{Conclusions}

Selective attention was impaired and information processing speed was slow in patients with thyrotoxic Graves' disease, and these findings were associated with age and educational level. After becoming euthyroid through antithyroid medication, these pathological findings returned to normal levels.

Review began 03/19/2021 Review ended 03/22/2021 Published 03/23/2021

(c) Copyright 2021

Ekinci et al. This is an open access article distributed under the terms of the Creative Commons Attribution License CC-BY 4.0., which permits unrestricted use, distribution, and reproduction in any medium, provided the original author and source are credited.
Additionally, Stroop interference effect was significantly decreased when patients with Graves' disease became euthyroid.

Categories: Endocrinology/Diabetes/Metabolism, Internal Medicine

Keywords: stroop interference effect, selective attention, graves' disease, information processing speed

\section{Introduction}

Graves' disease, the most common cause of hyperthyroidism, is an autoimmune disease associated with elevated circulating thyroid hormone levels [1]. Hyperactivity, palpitations, agitation, asthenia, heat intolerance, hand tremors, hyperreflexia, increased bowel movements, weight loss despite increased appetite, muscle weakness, irregular menstrual cycles, and mental problems can be observed due to elevated circulating thyroid hormone levels in patients with this condition [2].

Thyroid hormones play an important role in brain function. It has been reported that elevated thyroid hormone levels in hyperthyroidism caused oxidative stress, thereby leading to neuron damage, and patients with hyperthyroidism had decreased gray matter volume, wherein the cerebral metabolism and neurotransmitter system were affected by thyroid hormone levels [3]. Changes in the levels of neurotransmitters such as dopamine, which is associated with motivation; noradrenaline, which is associated with attention, concentration, and wakefulness; and serotonin, which is associated with mood, sleep, nutrition, and aggression may affect several neuropsychiatric functions, especially cognitive functions, thus leading to cognitive-behavioral pathologies in individuals with thyroid disease [4]. Therefore, these patients may easily get angry and exhibit mental pathologies such as irritability, anxiety, 
impulsiveness, emotional instability, inattention, inability to concentrate, sleeplessness, restlessness, and distractibility [5].

The Stroop Color and Word test, which is closely associated with frontal lobe functions, is a neuropsychological test that measures selective attention and information processing speed and analyzes cognitive processes [6]. In other words, this test evaluates the ability to inhibit cognitive interference that occurs when the processing of a particular stimulus feature prevents the simultaneous processing of a second stimulus feature. This test is based on the difference between the ink color in which a word is printed and the color expressed by the word. The ability to name the color despite the tendency to read is related to the ability and flexibility to shift attention and behavior [7]. Compared to the case when the ink color in which a word is printed and the color referred by the word are the same, if the said ink color is different from the color that the word refers to, the tendency to focus on naming the color and reading the name of the color causes a delay, which constitutes the Stroop interference effect [8].

This study aimed to (1) determine the selective attention level and information processing speed with the Stroop Color and Word test in patients recently diagnosed with thyrotoxic Graves' disease who had not yet started treatment, (2) observe the changes in attention levels and information processing speed when these patients became euthyroid under treatment, and (3) examine the relationship of selective attention level and information processing speed with thyroid hormone and autoantibody levels, as well as the psychopathological processes such as anxiety and depression.

\section{Materials And Methods}

\section{Study design}

This study was approved by the Ethics Committee of Health Sciences University, Kanuni Sultan Suleyman Training and Research Hospital (No: KAEK/2018.5.15). This prospective case-control study was conducted at the Internal Medicine Department of Health Sciences University, Kanuni Sultan Suleyman Training and Research Hospital. A total of 40 patients with Graves' disease and 40 age and gender-matched healthy controls were enrolled between June 1, 2018 and April 1, 2019.

\section{Patient characteristics and data collection}

The patient group comprised those who had thyrotoxicosis and were diagnosed with Graves' disease at the time of admission to the outpatient clinic, and the control group comprised healthy volunteers without any disease. Patients aged between 18 and 55 years, who were recently diagnosed with Graves' disease and were not yet started on antithyroid drug therapy, who had at least eight years of education, and who were coloraware and not colorblind were enrolled in the patient group. Patients who were illiterate, colorblind, or those who did not know colors; those aged $<18$ or $>55$ years; those with comorbidities other than Graves' disease; those with a known history of psychopathological or neurological disorders or those receiving treatment for such disorders; those with alcohol dependence; and those with a history of head trauma and any pathologies related to vision were excluded from the study.

Demographic data (age, gender, marital status, education level, and smoking status) of the subjects were recorded. The patient group was started on antithyroid drug therapy (methimazole) and followed up at the outpatient clinic at one-month intervals. Free T3 (fT3), free T4 (fT4), thyroid stimulating hormone (TSH), antithyroglobulin (antiTG), antithyroid peroxidase (antiTPO), and TSH receptor antibody (TRAb) levels were noted at the first admission visit when the patients had thyrotoxicosis and at the first visit when they became euthyroid.

\section{Stroop Color and Word test}

This test was performed in the patient group both at the visit when the patients had thyrotoxicosis and at the first visit when euthyroidism was observed, whereas the control group was subjected to the Stroop Color and Word test only at the visit conducted for inclusion in the study. The test comprised four white cards, each $14 \times 21.5 \mathrm{~cm}$ in size. Each card comprised six lines and each line contained four items. The test was applied in the following five stages:

Task one: Reading the names of colors printed in black ink on white background on the first card.

Task two: Reading the words, wherein the ink color is different from the color referred by the word.

Task three: Naming the colors of the circles printed in color.

Task four: Naming the colors of some words that are printed in color but do not refer to a color.

Task five: Naming the colors of words, wherein the ink color is different from the color referred by the word.

The time taken to complete each stage was measured by a stopwatch. The said time was evaluated as the 
time between the start of saying the first word or color and saying the last word or color on the card. The total time taken to complete all stages was obtained by adding up the times taken to complete each stage.

The number of errors and corrections made was noted for each stage. The total number of errors and corrections was calculated.

The first stage determines the level of reading speed, whereas the third and fourth stages show the speed of naming the color. The Stroop interference effect was calculated as the difference between the times taken to complete stages 5 and 1.

Beck Anxiety Inventory: This scale is a Likert-type, self-reported scale with 21 items scored between 0 and 3 [9]. The score range is $0-63$, and a high total score indicates higher severity of anxiety experienced by an individual.

Beck Depression Inventory: This scale comprised 21 items, 15 related to psychology and six related to somatic symptoms, with each item scored between 0 and 3 [10]. The highest score is 63, and a higher score indicates a higher level and severity of depression.

\section{Statistical analysis}

Statistical analysis was performed using SPSS for Windows 26.0 (IBM Corporation, Chicago, IL, USA). Categorical variables were expressed with numbers and percentages and numerical variables with mean \pm standard deviation values. Shapiro-Wilk test was used to determine how a variable is distributed. Chi-square test was used to compare categorical data between the groups. In comparing the two groups in terms of numerical variables, Student's t-test was used when the variables showed normal distribution, and MannWhitney U test when they showed non-normal distribution. Paired samples t-test or Wilcoxon test was used in the analysis of dependent quantitative data. Pearson's correlation analysis was performed for variables with normal distribution and Spearman correlation analysis for variables with non-normal distribution.

\section{Results}

Overall, 40 patients with Graves' disease and 40 healthy controls were included in the study. Both groups were similar in terms of mean age, gender distribution, marital status, smoking status, and education levels (Table 1). The scores obtained from Beck Anxiety Scale and Beck Depression Scale were higher in patients with thyrotoxic Graves' disease than in the control group, wherein the former also exhibited a higher incidence of anxiety and depression.

\begin{tabular}{|c|c|c|c|c|}
\hline & & Patient group & Control group & P-Value \\
\hline \multicolumn{2}{|l|}{ Age, years } & $34.67 \pm 11$ & $34.72 \pm 9.16$ & 0.983 \\
\hline \multirow[t]{2}{*}{ Gender } & Male, $\mathrm{n}$ & 16 & 19 & \multirow{2}{*}{0.499} \\
\hline & Female, $\mathrm{n}$ & 24 & 21 & \\
\hline \multirow[t]{2}{*}{ Marital status } & Married, n & 31 & 26 & \multirow{2}{*}{0.217} \\
\hline & Single, $\mathrm{n}$ & 9 & 14 & \\
\hline \multicolumn{2}{|l|}{ Smoker, n } & 18 & 15 & 0.496 \\
\hline \multicolumn{2}{|c|}{ Education time, year } & $11.7 \pm 4.4$ & $12.4 \pm 5.2$ & 0.492 \\
\hline \multicolumn{2}{|c|}{ Beck Anxiety Scale score } & $20 \pm 12.89$ & $10.8 \pm 6.44$ & $<0.001$ \\
\hline \multicolumn{2}{|c|}{ Presence of anxiety, n (\%) } & $22(55)$ & 9 (22.5) & 0.003 \\
\hline \multicolumn{2}{|c|}{ Beck Depression Scale score } & $17.55 \pm 10.05$ & $10.95 \pm 6.33$ & 0.001 \\
\hline \multicolumn{2}{|c|}{ Presence of depression, $n$ (\%) } & $17(42.5)$ & 7 (17.5) & 0.013 \\
\hline
\end{tabular}

TABLE 1: Demographic data of the groups.

The laboratory results at the first visit when patients with Graves' disease had thyrotoxicosis and at the visit when patients first became euthyroid are presented in Table 2. Free T3, fT4, antiTPO, antiTG, and TRAb levels significantly decreased, whereas TSH levels significantly increased in patients who became euthyroid under treatment. 


\section{Cureus}

\begin{tabular}{|c|c|c|c|}
\hline & Patient group, thyrotoxic status & Patient group, euthyroid status & P-Value \\
\hline $\mathrm{fT} 3, \mathrm{pg} / \mathrm{mL}$ & $11.43 \pm 7.02$ & $3.35 \pm 0.93$ & $<0.001$ \\
\hline $\mathrm{fT} 4, \mathrm{pg} / \mathrm{mL}$ & $3.37 \pm 1.85$ & $1.12 \pm 0.34$ & $<0.001$ \\
\hline $\mathrm{TSH}, \mathrm{ulU} / \mathrm{mL}$ & $0.01 \pm 0.02$ & $0.93 \pm 1.44$ & $<0.001$ \\
\hline AntiTPO, IU/mL & $249.71 \pm 204.49$ & $156.56 \pm 153.25$ & $<0.001$ \\
\hline AntiTG, IU/mL & $615.16 \pm 1081.02$ & $315.55 \pm 614.92$ & 0.007 \\
\hline TRAb, IU/mL & $9.14 \pm 11.18$ & $3.36 \pm 3.26$ & $<0.001$ \\
\hline
\end{tabular}

TABLE 2: Laboratory findings in Graves' disease.

fT3: free T3; fT4: free T4; TSH: thyroid stimulating hormone; AntiTPO: antithyroid peroxidase; AntiTG: antithyroglobulin; TRAb: TSH receptor antibody

Results of the Stroop Color and Word test performed during both the thyrotoxic and euthyroid periods in patients with Graves' disease and results of the Stroop Color and Word test of the control group are presented in Table 3. The completion times of the entire test as well as tasks 1, 2, and 5 had shortened; the number of self-corrections made in tasks 3, 4, and 5 and in the entire test had decreased; and the number of errors made in task 5 and the total number of errors made throughout the entire test had decreased when patients with thyrotoxic Graves' disease became euthyroid. The time taken to complete four cards except the second card and the time taken to complete the entire test were longer and the number of corrections made on the fifth card as well as the total number of corrections made during the entire test were higher in patients with thyrotoxic Graves' disease than in the control group. Comparing the patients with Graves' disease who were euthyroid with the control group, all results were similar except the time taken to complete task 1 . 


\begin{tabular}{|c|c|c|c|c|c|c|c|}
\hline & & Patient group, thyrotoxic status & Patient group, euthyroid status & p1 & Control group & p2 & p3 \\
\hline \multirow{3}{*}{ 1.task } & Errors & 0 & 0 & - & 0 & - & - \\
\hline & Self-corrections & 0 & 0 & - & 0 & - & - \\
\hline & Time, $\mathrm{s}$ & $11.58 \pm 4.44$ & $10.68 \pm 3.3$ & 0.003 & $8.99 \pm 1.5$ & 0.004 & 0.023 \\
\hline \multirow{3}{*}{ 2.task } & Errors & 0 & 0 & - & $0.02 \pm 0.15$ & - & - \\
\hline & Self-corrections & $0.15 \pm 0.42$ & $0.07 \pm 0.34$ & 0.317 & $0.1 \pm 0.3$ & 0.699 & 0.423 \\
\hline & Time, $\mathrm{s}$ & $11.89 \pm 4.2$ & $12.49 \pm 5.23$ & 0.572 & $10.77 \pm 2.87$ & 0.544 & 0.358 \\
\hline \multirow{3}{*}{ 3.task } & Errors & 0 & 0 & - & $0.02 \pm 0.15$ & - & - \\
\hline & Self-corrections & $0.17 \pm 0.44$ & $0.02 \pm 0.15$ & 0.034 & $0.12 \pm 0.33$ & 0.717 & 0.092 \\
\hline & Time, $\mathbf{s}$ & $11.24 \pm 3.4$ & $10.72 \pm 3.27$ & 0.054 & $9.63 \pm 1.74$ & 0.01 & 0.242 \\
\hline \multirow{3}{*}{ 4.task } & Errors & $0.1 \pm 0.63$ & $0.02 \pm 0.15$ & 0.655 & $0.05 \pm 0.22$ & 0.579 & 0.559 \\
\hline & Self-corrections & $0.7 \pm 1.09$ & $0.12 \pm 0.33$ & 0.001 & $0.3 \pm 0.46$ & 0.122 & 0.057 \\
\hline & Time, $\mathrm{s}$ & $20.37 \pm 7.48$ & $16.28 \pm 5.06$ & $<0.001$ & $15.23 \pm 4.17$ & $<0.001$ & 0.436 \\
\hline \multirow{3}{*}{ 5.task } & Errors & $0.65 \pm 1.29$ & $0.07 \pm 0.26$ & 0.002 & $0.2 \pm 0.4$ & 0.418 & 0.107 \\
\hline & Self-corrections & $1.7 \pm 1.6$ & $0.8 \pm 0.96$ & 0.001 & $0.62 \pm 0.97$ & 0.001 & 0.260 \\
\hline & Time, $\mathrm{s}$ & $27.73 \pm 8.92$ & $22.35 \pm 5.68$ & $<0.001$ & $20.91 \pm 5.31$ & $<0.001$ & 0.319 \\
\hline \multirow{3}{*}{ All tasks } & Errors & $0.75 \pm 1.46$ & $0.1 \pm 0.3$ & 0.004 & $0.32 \pm 0.65$ & 0.620 & 0.07 \\
\hline & Seli-corrections & $2.12 \pm 2.26$ & $1.02 \pm 1.16$ & $<0.001$ & $1.1 \pm 1.39$ & 0.001 & 0.915 \\
\hline & IIme, $\mathrm{s}$ & $82.84 \pm 24.51$ & $72.53 \pm 18.76$ & $<0.001$ & $65.55 \pm 13.69$ & 0.001 & 0.199 \\
\hline \multicolumn{2}{|c|}{ Stroop interference effect, s } & $16.14 \pm 7.29$ & $11.67 \pm 4.89$ & $<0.001$ & $11.91 \pm 5.31$ & 0.011 & 0.885 \\
\hline
\end{tabular}

TABLE 3: Comparison of the Stroop Color and Word Test results in thyrotoxic and euthyroid status of the patients with Graves' disease and control group.

p1: Wilcoxon test, p-value between "patient group, thyrotoxic status" and "patient group, euthyroid status"; p2: Mann-Whitney U test, p-value between "patient group, thyrotoxic status" and "control group"; p3: Mann-Whitney U test, p-value between "patient group, euthyroid status" and "control group"

Stroop interference effect significantly decreased when patients with Graves' disease became euthyroid. Stroop interference effect was significantly higher in patients with thyrotoxic Graves' disease than in the control group; however, there was no difference between the control group and euthyroid patients with Graves' disease in terms of Stroop interference. Gender (male: $14.54 \pm 4.65$, female: $17.21 \pm 8.55$; p: 0.557), smoking status (smoker: $18.28 \pm 7.93$, nonsmoker: $14.39 \pm 6.37$; p: 0.140 ), marital status (married: $16.43 \pm$ 8.04, single: $15.16 \pm 3.89 ; \mathrm{p}: 0.899$ ), presence of anxiety (patients with anxiety: $17.6 \pm 7.46$, those without anxiety: $14.36 \pm 6.85$ ), and presence of depression (patients with depression: $15.52 \pm 6.11$, those without depression: $16.6 \pm 8.16$; p: 0.957) did not show a significant difference in terms of Stroop interference effect in patients with thyrotoxic Graves' disease.

In the correlation analysis performed in patients with thyrotoxic Graves' disease, age and length of education had a significant correlation with Stroop interference effect and the time taken to complete the Stroop Color and Word test, the results of which are presented in Table 4. No significant correlation was observed in correlation analysis performed with the scores obtained from Beck Anxiety Scale, Beck Depression Scale, and all laboratory parameters (fT3, fT4, TSH, antiTPO, antiTG, and TRAb) in terms of the time taken to complete the Stroop Color and Word test and Stroop interference effect ( $p>0.05$, for all). 


\section{Cureus}

\begin{tabular}{|c|c|c|c|}
\hline & & Age, year & Education time, year \\
\hline \multirow[t]{2}{*}{ 1.task time, $\mathrm{s}$} & $r$ & 0.409 & -0.755 \\
\hline & $\mathrm{p}$ & 0.009 & $<0.001$ \\
\hline \multirow[t]{2}{*}{ 2.task tıme, $\mathrm{s}$} & $r$ & 0.345 & -0.747 \\
\hline & $\mathrm{p}$ & 0.029 & $<0.001$ \\
\hline \multirow[t]{2}{*}{ 3.task time, $\mathrm{s}$} & $r$ & 0.140 & -0.764 \\
\hline & $\mathrm{p}$ & 0.390 & $<0.001$ \\
\hline \multirow[t]{2}{*}{ 4.task time, $\mathrm{s}$} & $r$ & 0.209 & -0.888 \\
\hline & $\mathrm{p}$ & 0.195 & $<0.001$ \\
\hline \multirow[t]{2}{*}{ 5.task time, $\mathrm{s}$} & $r$ & 0.109 & -0.881 \\
\hline & $\mathrm{p}$ & 0.503 & $<0.001$ \\
\hline \multirow[t]{2}{*}{ All tasks time, $s$} & $r$ & 0.269 & -0.975 \\
\hline & $\mathrm{p}$ & 0.093 & $<0.001$ \\
\hline \multirow[t]{2}{*}{ Stroop interference, $\mathbf{s}$} & $r$ & -0.175 & -0.541 \\
\hline & $\mathrm{p}$ & 0.279 & $<0.001$ \\
\hline
\end{tabular}

TABLE 4: Correlation analysis between Stroop Color and Word Test findings and age and education time in thyrotoxic Graves' disease.

Spearman correlation analysis was performed

\section{Discussion}

In this study, selective attention was impaired and information processing speed was slowed in patients with thyrotoxic Graves' disease, but these pathological findings returned to normal levels when euthyroidism was achieved with treatment. The results of the Stroop Color and Word test were not associated with anxiety and depression in patients with Graves' disease who exhibited these psychopathologies more frequently than the healthy population. However, the results of this test were closely associated with age, especially with education level. In addition, thyroid hormone and autoantibody levels, gender, smoking, and marital status did not have a relationship with the results of the Stroop Color and Word test.

Patients with hyperthyroidism tended to have problems in decision-making under ambiguous conditions, and this pathology could stem from dopamine dysfunction and metabolic disorders in the frontal cortex and limbic system [4]. Changes in dopamine metabolism are thought to have negative effects on attention and concentration. In a study conducted with 27 patients with Graves' disease, attention and concentration tests were worse in the patient group than in the group with normal thyroid functions [11]. In the same study, it was also reported that elevated thyroid hormone levels could directly cause damage in attention pathways via an unknown mechanism, or the accelerated metabolism could lead to increased synthesis and secretion of various hormones that have an effect on attention and cause deterioration in attention and concentration in these patients. According to a study that examined various patient groups with thyroid dysfunction in terms of wakefulness, orientation, and executive control, negative outcomes were observed in terms of wakefulness in patients with hypothyroidism and in terms of wakefulness and executive control in patients with hyperthyroidism, whereas there were no negative outcomes in subclinical hypo and hyperthyroidism groups [12]. In the present study, parameters that assessed attention in the Stroop Color and Word test were worse in patients with Graves' disease, which is consistent with the literature.

The onset of dementia, a syndrome characterized by deterioration in memory and cognitive functions, was observed earlier in patients with hyperthyroidism, and suppressed TSH and elevated fT4 levels were also effective in the development of this syndrome [13]. In another study, overt hyperthyroidism, especially elevated fT4 levels, led to an increased risk of dementia [14]. In a study conducted with a geriatric patient group with low TSH levels and without dementia, low TSH levels were associated with poor cognitive functions and administration of methimazole slowed down cognitive function impairment [15]. Similarly, in the study by Jensovsky et al., normalization of the low TSH led to improved cognitive functions as well as improved verbal, visual, and general memory [16]. In the Stroop Color and Word test, which was used as a 
measure of cognitive functions in the present study, the aforementioned test results were worse (impaired) in the group of patients with Graves' disease than in the healthy population, whereas the aid impaired functions returned to normal levels when patients became euthyroid after antithyroid drug therapy.

In the study by Alvares et al., the tests related to attention and concentration were not associated with fT4 levels and free thyroxine index in patients with Graves' disease [11]. According to the study by Yuan et al., there was a negative correlation between executive control and fT4 in patients with hypothyroidism and a positive correlation between the same in patients with hyperthyroidism [12]. In our study, the parameters that measured attention and information processing speed did not have any relationship with thyroid hormone and autoantibody levels.

In the present study, anxiety and depression were more frequent in the group of patients with Graves' disease than in the healthy population, which is consistent with the literature $[5,12]$. More than half of our patient group had anxiety (55\%) and nearly half of the same group had depression (42.5\%). However, neither the presence of anxiety and depression nor the scores obtained from the tests that were performed for both psychopathologies showed any correlation with the results of the Stroop Color and Word test. There are several studies stating that these psychopathologies may have an effect on the Stroop effect; however, there are also studies reporting otherwise $[12,17]$.

Aging leads to some changes in brain structure and function, and these changes affect higher cognitive functions such as language processing and decision-making, especially the main cognitive functions such as attention and memory [18]. The time taken to perform any given task (such as the Stroop Color and Word test) in the presence of a distracting stimulus is longer in older adults than in young individuals. It is believed that the main reason for this prolonged response in older adults is a slowdown in information processing speed rather than decreased selective attention [18]. In the present study, there was a close relationship between age and the first two tasks in Stroop Color and Word test, whereas there was no relationship between Stroop interference effect and age.

Education is robustly associated with the level of cognitive function [19]. Early education, social learning experiences, and the skills necessary to pursue intellectual challenges throughout life contribute toward the development of cognitive functions [20]. In this study, there was a close relationship between education level and the parameters associated with selective attention and information processing speed. Moreover, response time in the test applied had shortened with a higher education level, and there was a significant correlation between the length of education and Stroop interference effect.

The first limitation of this study was the relatively small sample size. Another limitation was that the geriatric population was not included. Impairment in cognitive functions is an expected process in the geriatric patient group, and the extent to which these functions will be affected when Graves' disease is present may be a new study topic for researchers working in this field. Similarly, studies in which neurotransmitter levels affecting cognitive functions are measured and changes in brain tissue caused by elevated thyroid hormone levels are observed with cranial imaging may help clarify this issue.

\section{Conclusions}

In conclusion, this study showed that selective attention was impaired and information processing speed slowed down in patients with Graves' disease, but this negative effect disappeared when euthyroidism was achieved with treatment. The extent to which a person is affected by Graves' disease was closely associated with education level, but not with thyroid hormone or antibody levels. The number of relevant studies is very limited in the literature, and we think that our study has contributed toward the elucidation of this issue.

\section{Additional Information \\ Disclosures}

Human subjects: Consent was obtained or waived by all participants in this study. Ethics Committee of Health Sciences University, Kanuni Sultan Suleyman Training and Research Hospital issued approval KAEK/2018.5.15. Animal subjects: All authors have confirmed that this study did not involve animal subjects or tissue. Conflicts of interest: In compliance with the ICMJE uniform disclosure form, all authors declare the following: Payment/services info: All authors have declared that no financial support was received from any organization for the submitted work. Financial relationships: All authors have declared that they have no financial relationships at present or within the previous three years with any organizations that might have an interest in the submitted work. Other relationships: All authors have declared that there are no other relationships or activities that could appear to have influenced the submitted work.

\section{References}

1. De Leo S, Lee SY, Braverman LE: Hyperthyroidism. Lancet. 2016, 27:906-918. 10.1016/S0140- 
6736(16)00278-6

2. Subekti I, Pramono LA: Current diagnosis and management of Graves' disease . Acta Med Indones. 2018, 50:177-182.

3. Claustre J, Balende C, Pujol JF: Influence of the thyroid hormone status on tyrosine hydroxylase in central and peripheral catecholaminergic structures. Neurochem Int. 1996, 28:277-281. 10.1016/0197-018600088-7

4. Yuan L, Tian Y, Zhang F, Ma H, Chen X, Dai F, Wang K: Decision-making in patients with hyperthyroidism: a neuropsychological study. PLoS One. 2015, 19:129773. 10.1371/journal.pone.0129773

5. Fukao A, Takamatsu J, Arishima T, et al.: Graves' disease and mental disorders. J Clin Transl Endocrinol. 2019, 11:100207. 10.1016/j.jcte.2019.100207

6. Stroop JR: Studies of interference in serial verbal reactions . J Exp Psychol. 1935, 18:643-662. 10.1037/h0054651

7. MacLeod CM: The Stroop task: the "gold standard" of attentional measures . J Exp Psychol Gen. 1992, 121:1214. 10.1037/0096-3445.121.1.12

8. Glaser WR, Glaser MO: Context effects in Stroop-like word and picture processing . J Exp Psychol Gen. 1989, 118:13-42. 10.1037//0096-3445.118.1.13

9. Beck AT, Epstein N, Brown G, Steer RA: An inventory for measuring clinical anxiety: psychometric properties. J Consult Clin Psychol. 1988, 56:893-897. 10.1037//0022-006x.56.6.893

10. Beck AT, Ward CH, Mendelson M, Mock J, Erbaugh J: An inventory for measuring depression. Arch Gen Psychiatry. 1961, 4:561-571. 10.1001/archpsyc.1961.01710120031004

11. Alvares MA, Gomez A, Alavez E, Navarro D: Attention disturbance in Graves' disease. Psychoneuroendocrinology. 1983, 8:451-454. 10.1016/0306-4530(83)90026-4

12. Yuan L, Luan D, Xu X, et al.: Altered attention networks in patients with thyroid dysfunction: a neuropsychological study. Horm Behav. 2020, 121:104714. 10.1016/j.yhbeh.2020.104714

13. Joy Mathew C, Jose MT, Elshaikh AO, Shah L, Lee R, Cancarevic I: Is hyperthyroidism a possible etiology of early onset dementia?. Cureus. 2020, 22:10603. 10.7759/cureus.10603

14. George KM, Lutsey PL, Selvin E, Palta P, Windham BG, Folsom AR: Association between thyroid dysfunction and incident dementia in the atherosclerosis risk in communities neurocognitive study. J Endocrinol Metab. 2019, 9:82-89. 10.14740/jem588

15. Chachamovitz DS, Vigário Pdos S, Silva SO, Teixeira LB, Fagundes ML, Vaisman M, Teixeira Pde F: Does low-normal serum TSH level adversely impact cognition in elderly adults and might methimazole therapy improve outcomes?. Endocr J. 2016, 31:495-505. 10.1507/endocrj.EJ15-0458

16. Jensovský J, Spacková N, Hejduková B, Růzicka E: Effect of normalization of an isolated increase in TSH on the neuropsychological profile of patients [Article in Czech]. Cas Lek Cesk. 2000, 24:313-316.

17. Gerra G, Zaimovic A, Zambelli U, Timpano M, Reali N, Bernasconi S, Brambilla F: Neuroendocrine responses to psychological stress in adolescents with anxiety disorder. Neuropsychobiology. 2000, 42:82-92. $10.1159 / 000026677$

18. Murman DL: The impact of age on cognition . Semin Hear. 2015, 36:111-121. 10.1055/s-0035-1555115

19. Wilson RS, Hebert LE, Scherr PA, Barnes LL, Mendes de Leon CF, Evans DA: Educational attainment and cognitive decline in old age. Neurology. 2009, 3:460-465. 10.1212/01.wnl.0000341782.71418.6c

20. Parisi JM, Rebok GW, Xue QL, et al.: The role of education and intellectual activity on cognition . J Aging Res. 2012, 2012:416132. 10.1155/2012/416132 